\title{
Switchable Josephson junction based on interfacial exchange field
}

\author{
Cite as: Appl. Phys. Lett. 114, 022601 (2019); doi: 10.1063/1.5050382 \\ Submitted: 30 July 2018 - Accepted: 19 December 2018 - Published Online: \\ 14 January 2019
}

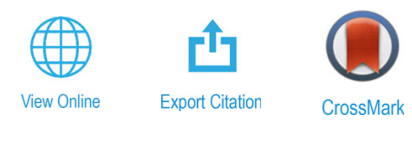

Juan Pedro Cascales, ${ }^{1,2, a)}$ Yota Takamura, ${ }^{1,2,3, b)}$ and Jagadeesh S. Moodera ${ }^{1,2,7, d)}$

\footnotetext{
AFFILIATIONS

a) Electronic mail: jcasc_87@mit.edu

b) Electronic mail: takamura@ee.e.titech.ac.jp

c) Electronic mail: sebastian_bergeret@ehu.eus

d) Electronic mail: moodera@mit.edu
}

${ }^{7}$ Francis Bitter Magnet Laboratory, Massachusetts Institute of Technology, Cambridge, Massachusetts 02139, USA

${ }^{2}$ Plasma Science and Fusion Center, Massachusetts Institute of Technology, Cambridge, Massachusetts 02139, USA

${ }^{3}$ School of Engineering, Tokyo Institute of Technology, Tokyo 152-8552, Japan

${ }^{4}$ Department of Physics, Northeastern University, Boston, Massachusetts 02115, USA

${ }^{5}$ Centro de Fisica de Materiales (CFM-MPC), Centro Mixto CSIC-UPV/EHU, Manuel de Lardizabal 4, E-20018 San Sebastian, Spain

${ }^{6}$ Donostia International Physics Center (DIPC), Manuel de Lardizabal 5, E-20018 San Sebastian, Spain

${ }^{7}$ Physics Department, Massachusetts Institute of Technology, Cambridge, Massachusetts 02139, USA

\begin{abstract}
We demonstrate exchange field switchable Josephson junctions where the Josephson tunneling between two superconducting layers $(\mathrm{Nb}$ and $\mathrm{NbN}$ ) could be controlled by the relative magnetic alignment of two GdN ferromagnetic insulator layers sandwiching the $\mathrm{Nb}$ layer. The junction's working principle is based on the control of the superconducting state of one of the layers by means of the interfacial exchange field of the magnetic GdN layers. At zero field and low temperatures, the ground state of the junctions corresponds to an antiferromagnetic configuration of the two GdN layers that coexist with the superconducting state of the Nb layer. By applying an external field, the GdN layers are switched to a parallel configuration, thereby suppressing the superconductivity in Nb and hence the Josephson current via interfacial exchange. This switchable Josephson junction may be useful for integrated superconducting spintronics and quantum circuits.
\end{abstract}

Published under license by AIP Publishing. https://doi.org/10.1063/1.5050382

The Josephson junction (JJ) is a versatile device which has found widespread application as field sensors, as measurement standards, or in quantum computing, among others. ${ }^{1-3}$ It consists of superconducting electrodes separated by a nonsuperconducting link (insulator, normal metal, etc) in which a (Josephson) current flows without dissipation.

There is particular interest in the study of Josephson junctions with magnetic interlayers. The interplay between the superconducting correlations and the exchange field in ferromagnetic materials leads to striking phenomena such as $0-\pi$ transition, ${ }^{4}$ long range triplet superconductivity, ${ }^{5}$ and anomalous Josephson currents. ${ }^{6}$ These phenomena and the ability of JJs to switch between super- and normal currents with high reproducibility and at high rates make magnetic Josephson junctions attractive for the emerging field of superconducting spintronics, ${ }^{7}$ building of cryogenic memories, ${ }^{8}$ and qubits for quantum computers. ${ }^{9}$

In this context, the combination of ferromagnetic insulators (FIs) and superconductors can be very useful. On the one hand, the strong interfacial exchange field (IEF) due to $s-d$ and $s-f$ exchange between the conducting electrons of the SC and the localized magnetic moments of the FIs leads to a full development of the triplet component ${ }^{5}$ and spin-dependent density of states. ${ }^{10-13}$ On the other hand, because there is no leakage of Cooper pairs into the FI, the superconducting gap is quite robust, and its magnitude is mainly controlled by the strength of the IEF via the paramagnetic effect. FI bilayers offer exceptionally high magnetic fields localized at atomically sharp 
interfaces. ${ }^{14}$ For a FI such as GdN, which involves the rare-earth element $\mathrm{Gd}$, the localized f electrons provide the IEF which in a superconductor can propagate over the superconducting coherence length. ${ }^{12}$ This exchange field is equivalent to a space inhomogeneous Zeeman field. By sandwiching a SC film between two such magnetic layers and keeping the SC layer's thickness below the coherence length, the effect of the exchange interactions at each interface sums up and can be greatly enhanced. The use of FIs has been recently shown to result in the passive control of superconducting or topological insulator layers in bilayers or spin valves. ${ }^{13-16}$ Moreover, an FI located between two superconducting electrodes may serve as a spin-filter, giving rise to spin-filtering Josephson junctions. ${ }^{12,17}$ Spin filtering Josephson junctions may exhibit a new type of $0-\pi$ transition, even pure triplet currents, ${ }^{18}$ and anomalous Josephson currents ( $\varphi_{0}$-junctions). ${ }^{19}$

In this letter, we report on the investigation of $\mathrm{FI} / \mathrm{SC} / \mathrm{FI} / \mathrm{SC}$ Josephson junctions with a built-in exchange driven magnetic switch. The proposed switchable Josephson junction device is based on the IEF induced in a superconductor sandwiched between two FI layers. In the FI/SC/FI structure, defined as a superconducting spin valve (SSV), the superconducting transition temperature $\mathrm{T}_{\mathrm{C}}$ of the $\mathrm{SC}$ spacer depends on the relative orientation of the magnetization of the two FI layers, ${ }^{20}$ referred to as the superconducting spin switch effect (SSE). ${ }^{14}$ We find that at zero field, the ground state corresponds to an AP configuration of the magnetization $\mathrm{M}$ in the FI layers. In this configuration, the net IEF in the superconductor between the FI layers cancels out and does not affect the superconductivity, allowing a Josephson current to flow. By applying a relatively small magnetic field, we can switch the two FI layers towards a parallel (P) alignment of magnetization. In this case, the average IEF experienced by the Cooper pairs destroys superconductivity in the SC spacer layer and consequently no supercurrent flows. Thus, the combination of the SSE effect and a FI tunneling barrier results in a passively controlled Josephson junction behavior that could be used as a non-volatile memory and logic for superconducting spintronics based on IEF. Moreover, our device could take us towards the full-triplet Josephson junction which would include a third FI layer. ${ }^{18}$

The samples were fabricated by reactive sputtering in an ultra-high vacuum (UHV) system on thermally oxidized Si substrates at room temperature starting with a $1 \mathrm{~nm}$ thick seed layer of $\mathrm{Al}_{2} \mathrm{O}_{3}$. As shown in Fig. 1(a), the layer sequence of the sample is $\operatorname{AlN}(5) / \mathrm{NbN}(10) / \mathrm{GdN}(3) / \mathrm{Nb}(10) / \mathrm{GdN}(3) / \mathrm{AlN}(1) / \mathrm{Al}(20)$ with the numbers in parentheses corresponding to the thickness in $\mathrm{nm}$. In these structures, the $\mathrm{SC}$ electrodes $(\mathrm{NbN}$ and $\mathrm{Nb}$ ) are separated by a $3 \mathrm{~nm}$ GdN tunnel barrier. The second GdN layer situated over the $\mathrm{Nb}$ layer, together with the tunneling barrier, sandwiches the $\mathrm{Nb}$ layer allowing us to control its superconducting state via the relative magnetic configuration (P or AP) of the GdN magnetic moments. The optimum thicknesses of both GdN layers were arrived at by systematically fabricating junctions with different $\mathrm{Nb}$ and $\mathrm{GdN}$ thicknesses, as well as by different growth conditions, until Josephson tunneling was observed. The combination of a thin AlN layer followed by a thick Al layer was found to be the best capping to protect samples from degradation while preserving vertical transport.
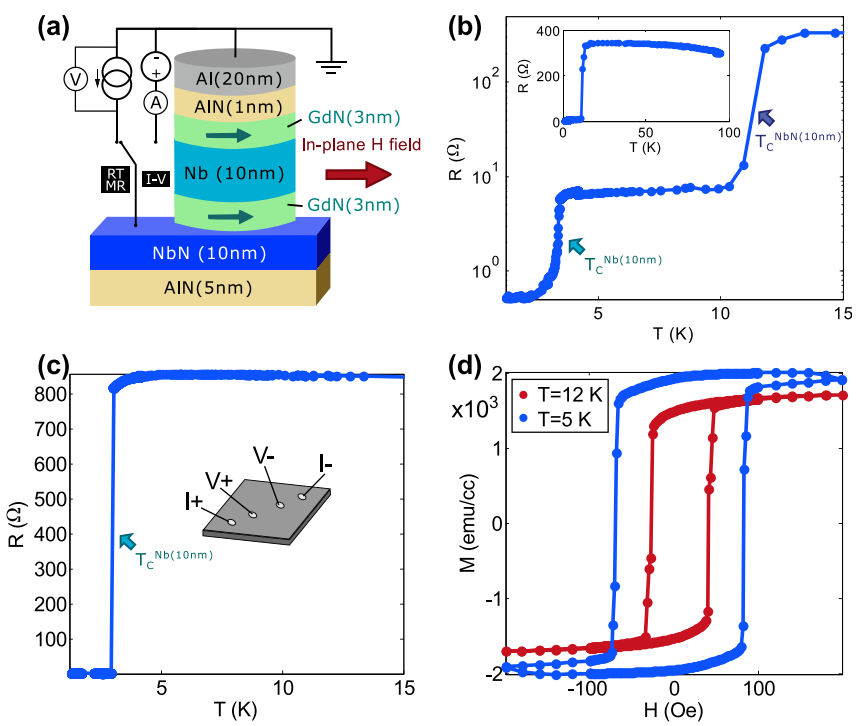

FIG. 1. (a) Schematic of the sample structure and transport measurements. The junctions are circular pillars of diameters ranging from 10 to $80 \mu \mathrm{m}$. (b) Resistance vs temperature at zero field (ZFC) of a $10 \mu \mathrm{m}$ device at a current bias of $10 \mu \mathrm{A}$. Two drops are observed at $T=11.3 \mathrm{~K}$ and $T=3.2 \mathrm{~K}$, corresponding to the superconducting critical temperature of the $10 \mathrm{~nm} \mathrm{NbN}\left(T_{C}^{N b N(10)}\right)$ and $10 \mathrm{~nm} \mathrm{Nb}\left(T_{C}^{N b(10)}\right)$ films, respectively. The inset depicts $R$ vs $T$ up to higher temperature. (c) $R$ vs $T$ (ZFC) of an unpatterned layered structure in which only the $\mathrm{Nb}$ film has been contacted, with $T_{C}=3 \mathrm{~K}$. (d) $\mathrm{M}-\mathrm{H}$ loops for the unpatterned sample at 5 and $12 \mathrm{~K}$, showing only one coercive field for the GdN sandwich.

After deposition, the samples were patterned by optical lithography and ion milling into circular junctions with diameters ranging from 10 to $80 \mu \mathrm{m}$. The milling was carried out down to the bottom $\mathrm{NbN}$ layer, leaving it intact. The pillar structure was protected by $20 \mathrm{~nm}$ of $\mathrm{Al}_{2} \mathrm{O}_{3}$ filler, and a $100 \mathrm{~nm}$ thick $\mathrm{Al}$ contact pad of $1 \mathrm{~mm}^{2}$ area was fabricated through an additional lithography/sputtering step. Independent electrical contact with indium was made to each SC layer via the top electrode pad $(\mathrm{Nb})$ and by scratching a corner of the wafer to expose $\mathrm{NbN}$.

Figure 1(b) plots resistance vs temperature, zero-field cooled (ZFC), of a $10 \mu \mathrm{m}$ device (essentially a 3-terminal measurement), with an applied current $\mathrm{I}=10 \mu \mathrm{A}$. Two clear drops in resistance occur at the superconducting transition temperatures of the $10 \mathrm{~nm} \mathrm{NbN}$ layer $\left(\mathrm{T}_{\mathrm{C}}^{\mathrm{NbN}(10)}=11.3 \mathrm{~K}\right)$ and the $10 \mathrm{~nm} \mathrm{Nb}$ layer $\left(\mathrm{T}_{\mathrm{C}}^{\mathrm{Nb}(10)}=3.2 \mathrm{~K}\right)$, where each $\mathrm{T}_{\mathrm{C}}$ was taken as the mid-point of each resistive transition. The reduced value of $\mathrm{T}_{\mathrm{C}}$ of the $\mathrm{Nb}$ layer (up to $7.5 \mathrm{~K}$ for pure $10 \mathrm{~nm} \mathrm{Nb}^{21}$ ) is mainly credited to the proximity of the magnetic layers. The highest resistance value achieved before the first SC transition is $343 \Omega$. The drop from 343 to $7 \Omega$ when NbN becomes SC can be accounted for in order of magnitude by the spread resistance in the $\mathrm{NbN}$ layer experienced between the scratched indium contact and the bottom pillar. When the SC transition of the Nb layer takes place, the resistance further drops down to $\sim 0.5 \Omega$, which we attribute to the occurrence of Josephson tunneling. The residual $0.5 \Omega$ resistance could be ascribed to a rougher top AlN/GdN layer. Figure 1(c) shows R vs T (ZFC) of the $10 \mathrm{~nm} \mathrm{Nb}$ film in an unpatterned multilayer (only the $\mathrm{Nb}$ film was contacted), in which the critical 
temperature is $0.2 \mathrm{~K}$ lower than that for the patterned devices $\left(\mathrm{T}_{\mathrm{Cb}}^{\mathrm{Nb}(10)}=3.0 \mathrm{~K}\right)$, which could be due to a larger IEF from the unpatterned magnetic films. Figure 1 (d) presents magnetic hysteresis data obtained by SQUID of the unpatterned structure at $\mathrm{T}=5 \mathrm{~K}$ and $\mathrm{T}=12 \mathrm{~K}$. The coercive field value is the same for both GdN films and it increases as temperature is lowered. In other words, at temperatures above the $\mathrm{Nb} \mathrm{SC}$ transition, the FI sandwich is always in the P state.

The patterned sample was then warmed up above $\mathrm{T}_{\mathrm{C}}^{\mathrm{Nb}(10)}$ and cooled again while recording magnetoresistance (MR) curves with $\mathrm{I}=10 \mu \mathrm{A}$ and an in-plane magnetic field. A residual resistance in the $\mathrm{AP}$ state at the lowest temperature of $\mathrm{R}_{\text {res.1.3K }}^{\mathrm{AP}}$ $=0.05 \Omega$ was observed and subtracted from all measurements shown in Figs. 2(a) and 2(b) for simplicity of analysis. As shown in Fig. 2(a), when the temperature reaches the superconducting transition of the Nb layer, the MR signal exhibits sharp switching between low and high resistance states. We attribute this change in resistance to result from the AP (low resistance) and $\mathrm{P}$ (high resistance) alignment of the GdN magnetizations, respectively, according to the spin switching effect. ${ }^{14,15,20}$ No MR signal was observed for higher temperatures.

We note that the low resistance or $R_{A P}$ state appears for small or no applied magnetic field. The MR loop is symmetric with respect to the field, and the low resistance region widens so the switching field value $\left(\mathrm{H}_{\text {switch }}\right)$ increases as the temperature decreases. Additionally, there is some hysteresis in $H_{\text {switch }}$ as the values are 12.5 Oe higher on average for an increasing field sweep than for decreasing (see Fig. S.6 in the supplementary material).

Figure 2(b) plots resistance vs temperature at constant applied magnetic field, extracted from the MR data in Fig. 2(a). The curves exhibit a sharp jump from a high resistance state $\left(R_{P}\right)$ to a low resistance state $\left(R_{P}\right)$ at progressively lower temperatures as with increasing field.

We describe $R_{A P}$ and $R_{P}$ by the equation ${ }^{22} R(T)=\frac{R_{N}}{2}[1$ $\left.+\tanh \frac{T-T_{C}}{\delta T}\right]+R_{\text {offset }}$, where $R_{N}$ is the normal state resistance, $T_{C}$ is the critical temperature, $\delta \mathrm{T}$ is the width of the transition, and $R_{\text {offset }}$ is used to describe the $R_{P}$ data at the lowest temperature. There is no offset resistance for $R_{A P}$, while $R_{\text {offset }}^{P}=0.34 \Omega$, which could be due to the stray-field (or dipolar coupling) between the two GdN layers suppressing superconductivity in the Nb layer.
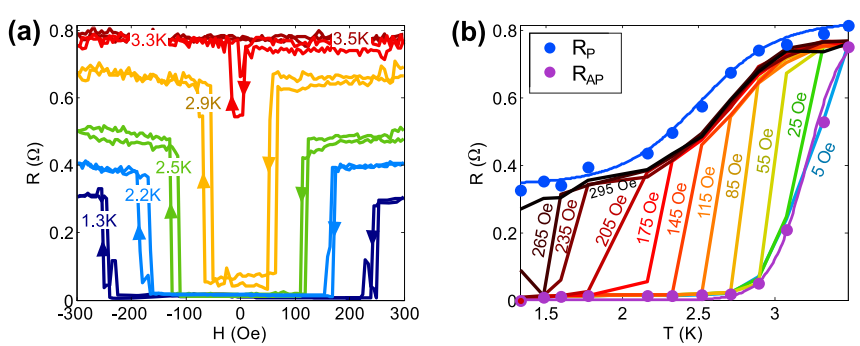

FIG. 2. (a) MR of a $10 \mu \mathrm{m}$ device at different temperatures, exhibiting sharp switching between low and high resistance states. The curves are symmetric with field and are attributed to the SSE and an antiferromagnetic coupling between the GdN films through the superconducting Nb layer. ${ }^{15,20}$ (b) Resistance vs temperature at different magnetic fields, constructed from the data in (a). The curves exhibit a sharp transition from a high $\left(R_{P}\right)$ to a low resistance state $\left(R_{A P}\right)$, which show a maximal difference in $T_{C}$ of $\Delta T_{C} \sim 0.7 \mathrm{~K}$ due to the SSE.
We obtain $\mathrm{T}_{\mathrm{C}}^{\mathrm{AP}}=3.22 \mathrm{~K}$ and $\mathrm{T}_{\mathrm{C}}^{\mathrm{P}}=2.5 \mathrm{~K}$, yielding a difference of $\Delta \mathrm{T}_{\mathrm{C}} \sim 0.7 \mathrm{~K}$, and a widening of the $\mathrm{SC}$ transition $\left(\delta \mathrm{T}^{\mathrm{AP}}=0.24 \mathrm{~K}\right.$ and $\delta \mathrm{T}^{\mathrm{AP}}=0.52 \mathrm{~K}$ ) due to the different IEFs induced by the GdN layers for both magnetic configurations.

The fact that the value of $\mathrm{H}_{\text {switch }}$ increases as temperature is lowered could be, at first glance, ascribable to the behavior for the upper critical field $\mathrm{H}_{\mathrm{C} 2}(\mathrm{~T})$. However, there are several features of our observations that do not support such assumptions: First, superconductors exhibit a smooth decrease in $\mathrm{T}_{\mathrm{C}}$ and a broadening of the transition as field is increased, as opposed to sharp switching observed here. As shown in the supplementary material, we model the expected MR for our $\mathrm{Nb}$ layer by using the $R(T)$ equation ${ }^{22}$ and by linearly decreasing $T_{C}$ from $T_{C}^{A P}$ to $T_{C}^{P}$ while increasing $\delta \mathrm{T}$ from $\delta \mathrm{T}^{\mathrm{AP}}$ to $\delta \mathrm{T}^{\mathrm{P}}$. While the obtained curves are symmetric with $\mathrm{H}$ and the low resistance region widens with decreasing temperature, they do not account for the experimentally observed sharp, square-like switching. Second, we clearly observe a hysteresis in $\mathrm{H}_{\text {switch }}$. Finally, assuming that $\mathrm{H}_{\text {switch }}$ is related to the upper critical field, such values are at least an order of magnitude too low for a $10 \mathrm{~nm} \mathrm{Nb}$ film. ${ }^{23}$

Based on all these, we attribute the sharp switching in MR curves, the temperature dependence and hysteresis of $\mathrm{H}_{\text {switch }}$, the reduced critical temperature of $\mathrm{Nb}$, and the resistance of $R_{A P}$ at low temperatures to the internal exchange field due to the proximity coupling with GdN layers in the sandwich ${ }^{14,15,20}$ and an increasing antiferromagnetic (AF) coupling between FIs taking place through the $\mathrm{SC} \mathrm{Nb}$ film as the temperature is lowered. ${ }^{15,20}$

The appearance of an AF coupling has been previously reported in $\mathrm{GdN} / \mathrm{Nb} / \mathrm{GdN}$ trilayers for temperatures below the $\mathrm{SC}$ transition of the Nb spacer. ${ }^{15}$ As can be seen in our GdN/Nb/ GdN trilayer control samples in the supplementary material, solely symmetric MR curves can be obtained for certain GdN and $\mathrm{Nb}$ thicknesses, as was seen for our JJ devices.

The SC transition temperature of the sandwiched $\mathrm{Nb}$ layer, the AF coupling strength, and its influence on the MR signal depend on the absolute and relative thicknesses of the GdN layers and the $\mathrm{Nb}$ spacer. ${ }^{15}$ For different $\mathrm{GdN}$ and $\mathrm{Nb}$ thicknesses, the appearance of the AF coupling can be shifted further below the SC transition temperature so both remanent AP and P states can be obtained for some range of temperatures, which is desired for technological applications. However, in the case of our JJs, the thickness of the layers for which we achieve Josephson tunneling yields an AF coupling which prevents obtaining both remanent $\mathrm{P}$ and AP states.

We also determined the current-voltage (I-V) characteristics of our JJs by sweeping voltage and measuring the current through the device. Figure 3(a) plots I-V curves for the $10 \mu \mathrm{m}$ junction at different temperatures and magnetic field values. As expected, for temperatures above $\mathrm{T}_{\mathrm{C}}^{\mathrm{Nb}(10)}$, the junction presents no sign of Josephson current. However, at $1.3 \mathrm{~K}$ and zero-field, the junction presents a clear Josephson effect with a critical current of around $140 \mu \mathrm{A}$. Furthermore, if we use Fig. 2(a) as a guide, at $1.3 \mathrm{~K}$, the zero-voltage state remains up to fields \pm 250 Oe. In fact, the curve shown in Fig. 3(a) for 200 Oe differs very little from the I-V obtained with no applied field, whereas for 300 Oe, the critical current is strongly suppressed and the I-V 
characteristics are closer to those of the normal state I-Vs (above $3.3 \mathrm{~K}$ ). We ascertained that negative or positive fields as well as the magnetic history do not influence the I-Vs up to around 230 Oe at $1.3 \mathrm{~K}$. For brevity, only positive field results are shown.

Figure 3(b) plots the dependence of the Josephson critical current $\mathrm{I}_{C}$ on the applied in-plane field (step size between 1 and $3 \mathrm{Oe}$ ) at $\mathrm{T}=1.3 \mathrm{~K}$. $\mathrm{I}_{\mathrm{C}}$ is extracted at each field by fitting the I-V

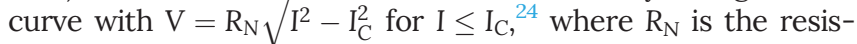
tance of the junction in the normal state (see supplementary material). The inset of Fig. 3(b) shows the full range, where a sharp drop in critical current takes place at the switching field (around 250 Oe) between the AP and P states. As shown in Fig. $3(\mathrm{c})$, the $\mathrm{I}_{\mathrm{C}}$ oscillations at low fields can be compared to the Airy pattern, theoretically expected for a circular junction. ${ }^{3,25} I_{C}$ for fields below 60 Oe from Fig. 3(b) is normalized between 0 and 1 for simplicity and fit using the equation $\left|2 \frac{J_{1}(x)}{x}\right|$, where $J_{1}$ is a Bessel function of first kind and $x=2 \pi\left(\mathrm{H}-\mathrm{H}_{\mathrm{off}}\right) / \mathrm{H}_{0}$, where $\mathrm{H}_{\text {off }}$ is the shift in the field of the Fraunhofer pattern ${ }^{17}$ and $\mathrm{H}_{0}$ is the oscillation period. The results of the fitting are $\mathrm{H}_{\mathrm{O}}=14.9$ and $\mathrm{H}_{\text {off }}$ $=3.8 \mathrm{Oe}$. If we now take this value of $\mathrm{H}_{0}$ and we consider that the flux through the junction obeys $\phi_{0}=(2 \lambda+t) d \mu_{0} H_{0},{ }^{26}$ where $\phi_{0}$ is the magnetic flux quantum, $t=3 \mathrm{~nm}$ the junction barrier thickness, and $d=10 \mu \mathrm{m}$ the junction diameter, we can estimate $\lambda$ the average field penetration depth of the junction. We obtain $\lambda=68 \mathrm{~nm}$, which is a reasonable result as it is an intermediate value of $\lambda$ for the two different materials used as SC electrodes $\left[\lambda_{\mathrm{Nb}}=39 \mathrm{~nm}^{27}\right.$ and $\lambda_{\mathrm{NbN}}=194 \mathrm{~nm}$ (Ref. 28)]. Although the $\mathrm{I}_{\mathrm{C}}(\mathrm{H})$ dependence is far from a Fraunhofer pattern, some conclusions can be extracted if one zooms in the low-field region and $\mathrm{I}_{C}$ normalized [Fig. 3(c)]. First, one notes that the maximum is shifted
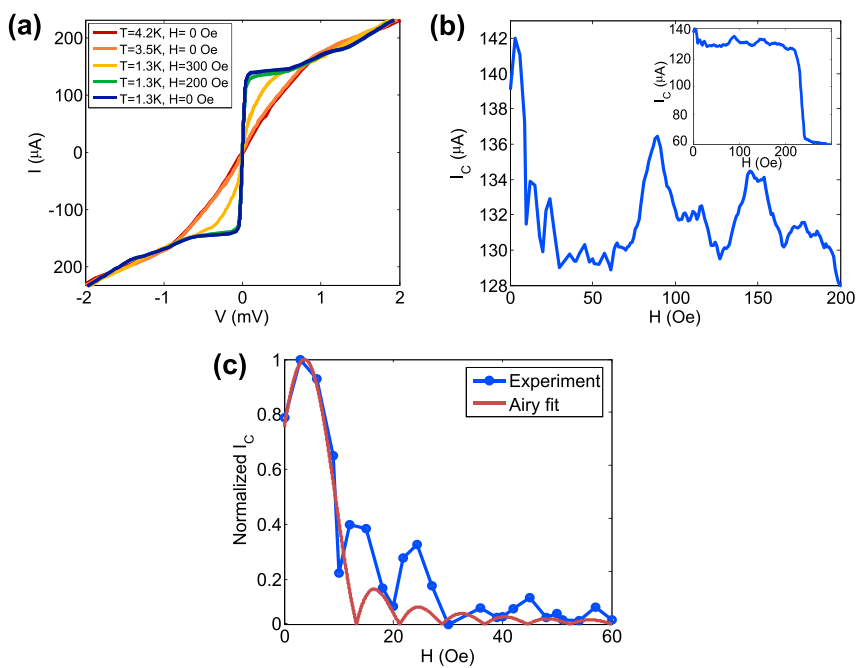

FIG. 3. (a) Current-voltage plots at different temperatures and applied magnetic fields for a $10 \mu \mathrm{m}$ Josephson junction, with a maximum critical current $I_{C}=142$ $\mu \mathrm{A}$. (b) Field dependence of $I_{C}$ at $T=1.3 \mathrm{~K}$ showing oscillations at fields below 200 Oe. The inset shows how $I_{C}$ sharply drops at 2500 e when the junction switches from the AP to the P state. (c) Fit of the Airy pattern expected for circular Josephson junctions to the normalized $I_{C}$ at low fields, yielding a field period of $H_{0}$ $=14.9$ Oe and an offset of $H_{\text {off }}=3.80$ e. from zero field, which is a manifestation of the finite magnetization of the barrier GdN layer between the superconductors. ${ }^{29}$ Second, although one distinguishes after normalization of the curve [see Fig. 3(c)] an oscillation of $I_{C}$ that resembles the Airy pattern expected for circular Josephson junctions, ${ }^{3}$ this pattern is distorted when the applied field gets larger. Moreover, the minima of $I_{C}$ do not vanish at any field. These observations may be attributed to the highly asymmetric distribution of the current density through the junction. The asymmetry is field dependent: At very low fields, the junction is in a AF configuration, and one can assume that magnetic disorder is low. This may explain the "Fraunhofer-like" behavior of the $I_{C}(H)$ curve in Fig. 3(c) for $\mathrm{H}<40$ Oe. As the applied magnetic field becomes higher, it does not only affect the spatial behavior of the superconducting phase but also the magnetic configuration of the ferromagnetic layers. At higher field, the AF configuration becomes unstable and the GdN layers tend towards the P configuration and thus form a complex domain structure. Furthermore, in a multi-domain state, stray fields from the domain walls lead to a highly non-uniform superconducting order parameter, hence to a random interference pattern, and to the highly nonuniform current density. ${ }^{29,30}$ This asymmetry could cause the nonvanishing minima of $\mathrm{I}_{\mathrm{C}}{ }^{3}$ The non-zero Josephson current in the AP state (fields above 250 Oe) is a result of the fitting, since it can be argued there is no Josephson current flowing from the curvature of the IV curve at $\mathrm{T}=1.3 \mathrm{~K}$ and $\mathrm{H}=300$ Oe.

In conclusion, we have fabricated Josephson junction structures which incorporate an internal exchange-driven magnetic switch based on the superconducting spin switching effect. The use of ferromagnetic insulator layers to sandwich one of the superconducting electrodes of the junction, with one of the FI layers additionally acting as the JJ tunneling barrier, allows the control of the superconducting state of the sandwiched SC electrode through the interfacial exchange field. This control of the superconducting state results in a fully switchable Josephson junction, between superconductor-insulator-superconductor and superconductor-insulator-normal metal tunneling in the absence of an applied magnetic field. The vertical geometry of our device opens an opportunity to integrate such valves into superconducting circuits in analogy to magnetic tunnel junctions. ${ }^{31}$ Moreover, by adding an additional FI layer in the same setup, one may be able to realize $\pi$ - and $\varphi_{0}$-junctions predicted in recent works. ${ }^{18,19}$ Different avenues will be explored to obtain non-volatile magnetic states to widen the potential applications of our device: further optimizing the $\mathrm{GdN}$ and $\mathrm{Nb}$ system, e.g., employing ultrathin FI layers to obtain out-of-plane anisotropies; varying the pillar geometry to introduce shape anisotropies and hard magnetic axes; and testing other superconducting materials with higher Tc as the core of the switch such as $\mathrm{NbN}$ and $\mathrm{MgB}_{2}$.

See supplementary material for details about additional Josephson junction measurements, GdN/Nb/GdN control sample measurements, simulation of the effect of an external field on $\mathrm{MR}$, and fitting equation to extract critical current from I-V plots.

This work was supported by NSF Grant No. DMR-1700137 and ONR Grant No. N00014-16-1-2657. J.P.C. acknowledges 
support from the Fundacion Seneca (Region de Murcia) postdoctoral fellowship (19791/PD/15). Y.T. acknowledges support from JSPS Postdoctoral Fellowships for Research Abroad. The work of G.S. and D.H. was funded by the National Science Foundation Grant No. ECCS-1402738. The work of F.S.B. was supported by the Spanish Ministerio de Economia, Industria y Competitividad under Project Nos. FIS2014-55987P and FIS2017-82804-P. F.S.B. acknowledges funding from the EU's Horizon 2020 research and innovation programme under grant agreement No. 800923 (SUPERTED).

\section{REFERENCES}

${ }^{\mathbf{1} B .}$ Josephson, Phys. Lett. 1, 251 (1962).

${ }^{2}$ B. D. Josephson, Rev. Mod. Phys. 46, 251 (1974).

${ }^{3}$ A. Barone and G. Paterno, Physics and Applications of the Josephson Effect (Wiley Online Library, 1982), Vol. 1.

${ }^{4}$ A. I. Buzdin, Rev. Mod. Phys. 77, 935 (2005).

${ }^{5}$ F. Bergeret, A. F. Volkov, and K. B. Efetov, Rev. Mod. Phys. 77, 1321 (2005).

${ }^{6}$ A. Buzdin, Phys. Rev. Lett. 101, 107005 (2008).

${ }^{7}$ M. Eschrig, Phys. Today 64(1), 43 (2011); J. Linder and J. W. Robinson, Nat. Phys. 11, 307 (2015).

${ }^{8}$ E. Gingrich, B. M. Niedzielski, J. A. Glick, Y. Wang, D. Miller, R. Loloee, W. Pratt, Jr., and N. O. Birge, Nat. Phys. 12, 564 (2016).

${ }^{9}$ A. K. Feofanov, V. A. Oboznov, V. V. Bol'Ginov, J. Lisenfeld, S. Poletto, V. V. Ryazanov, A. N. Rossolenko, M. Khabipov, D. Balashov, A. B. Zorin et al., Nature Phys. 6, 593 (2010).

${ }^{10}$ X. Hao, J. S. Moodera, and R. Meservey, Phys. Rev. B 42, 8235 (1990).

"B. Li, G.-X. Miao, and J. S. Moodera, Phys. Rev. B 88, 161105 (2013).

${ }^{12}$ G. -X. Miao and J. S. Moodera, Phys. Chem. Chem. Phys. 17, 751 (2015).
${ }^{13}$ E. Strambini, V. N. Golovach, G. De Simoni, J. S. Moodera, F. S. Bergeret, and F. Giazotto, Phys. Rev. Mater. 1, 054402 (2017).

${ }^{14}$ B. Li, N. Roschewsky, B. A. Assaf, M. Eich, M. Epstein-Martin, D. Heiman, M. Münzenberg, and J. S. Moodera, Phys. Rev. Lett. 110, 097001 (2013).

${ }^{15}$ Y. Zhu, A. Pal, M. G. Blamire, and Z. H. Barber, Nat. Mater. 16, 195 (2017).

${ }^{16} \mathrm{M}$. Li, W. Cui, J. Yu, Z. Dai, Z. Wang, F. Katmis, W. Guo, and J. Moodera, Phys. Rev. B 91, 014427 (2015).

${ }^{17}$ K. Senapati, M. G. Blamire, and Z. H. Barber, Nat. Mater. 10, 849 (2011).

${ }^{18}$ F. S. Bergeret, A. Verso, and A. F. Volkov, Phys. Rev. B 86, 060506 (2012); F. Bergeret, A. Verso, and A. F. Volkov, Phys. Rev. B 86, 214516 (2012).

${ }^{19}$ M. A. Silaev, I. V. Tokatly, and F. S. Bergeret, Phys. Rev. B 95, 184508 (2017).

${ }^{20}$ P. D. Gennes, Phys. Lett. 23, 10 (1966).

${ }^{21}$ C. Delacour, L. Ortega, M. Faucher, T. Crozes, T. Fournier, B. Pannetier, and V. Bouchiat, Phys. Rev. B 83, 144504 (2011).

${ }^{22}$ J. A. Burney, Ph.D. thesis, Stanford University, 2007.

${ }^{23}$ J. Quateman, Phys. Rev. B 34, 1948 (1986).

${ }^{24}$ J. A. Glick, M. A. Khasawneh, B. M. Niedzielski, R. Loloee, W. P. Pratt, N. O. Birge, E. C. Gingrich, P. G. Kotula, and N. Missert, J. Appl. Phys. 122, 133906 (2017).

${ }^{25}$ R. L. Peterson and J. W. Ekin, Phys. Rev. B 42, 8014 (1990).

${ }^{26} \mathrm{M}$. Tinkham, Introduction to Superconductivity (Courier Corporation, 1996).

${ }^{27}$ B. W. Maxfield and W. L. McLean, Phys. Rev. 139, A1515 (1965).

${ }^{28}$ B. Komiyama, Z. Wang, and M. Tonouchi, Appl. Phys. Lett. 68, 562 (1996).

${ }^{29}$ T. S. Khaire, W. P. Pratt, and N. O. Birge, Phys. Rev. B 79, 094523 (2009).

${ }^{30}$ M. A. Khasawneh, T. S. Khaire, C. Klose, W. P. Pratt, Jr., and N. O. Birge, Supercond. Sci. Technol. 24, 024005 (2011).

${ }^{31}$ J. S. Moodera, L. R. Kinder, T. M. Wong, and R. Meservey, Phys. Rev. Lett. 74, 3273 (1995). 